\title{
Leptin induces fasting hypoglycaemia in a mouse model of diabetes through the depletion of glycerol
}

\author{
Heather C. Denroche • Michelle M. Kwon • Whitney L. Quong • \\ Ursula H. Neumann • Jerzy E. Kulpa • Subashini Karunakaran • \\ Susanne M. Clee $\cdot$ Roger W. Brownsey $\cdot$ Scott D. Covey • \\ Timothy J. Kieffer
}

Received: 16 September 2014 / Accepted: 28 January 2015 / Published online: 26 February 2015

(C) Springer-Verlag Berlin Heidelberg 2015

\begin{abstract}
Aims/hypothesis Leptin has profound glucose-lowering effects in rodent models of type 1 diabetes, and is currently being tested clinically to treat this disease. In addition to reversing hyperglycaemia, leptin therapy corrects multiple lipid, energy and neuroendocrine imbalances in rodent models of type 1 diabetes, yet the precise mechanism has not been fully defined. Thus, we performed metabolic analyses to delineate the downstream metabolic pathway mediating leptin-induced glucose lowering in diabetic mice.

Methods Mice were injected with streptozotocin (STZ) to induce insulin-deficient diabetes, and were subsequently treated with $20 \mu \mathrm{g} /$ day recombinant murine leptin or vehicle for 5 to 14 days. Energy-yielding substrates were measured in the
\end{abstract}

Electronic supplementary material The online version of this article (doi:10.1007/s00125-015-3529-4) contains peer-reviewed but unedited supplementary material, which is available to authorised users.

H. C. Denroche • M. M. Kwon • W. L. Quong • U. H. Neumann • T. J. Kieffer $(\square)$

Laboratory of Molecular and Cellular Medicine, Department of

Cellular and Physiological Sciences, Life Sciences Institute,

University of British Columbia, 2350 Health Sciences Mall,

Vancouver, BC, Canada V6T 1Z3

e-mail: tim.kieffer@ubc.ca

J. E. Kulpa $\cdot$ R. W. Brownsey $\cdot$ S. D. Covey

Department of Biochemistry and Molecular Biology, University of

British Columbia, Vancouver, BC, Canada

S. Karunakaran $\cdot$ S. M. Clee

Laboratory of the Genetics of Obesity and Diabetes, Department of Cellular and Physiological Sciences, University of British Columbia,

Vancouver, BC, Canada

\section{T. J. Kieffer}

Department of Surgery, University of British Columbia,

Vancouver, BC, Canada liver and plasma, and endogenous glucose production was assessed by tolerance to extended fasting.

Results STZ-leptin-treated mice developed severe hypoketotic hypoglycaemia during prolonged fasting, indicative of suppressed endogenous ketone and glucose production. STZ-leptin mice displayed normal gluconeogenic and glycogenolytic capacity, but had depleted circulating glycerol and NEFA. The depletion of glycerol and NEFA correlated tightly with the kinetics of glucose lowering in response to chronic leptin administration, and was not mimicked by single leptin injection. Administration of glycerol acutely reversed fasting-induced hypoglycaemia in leptin-treated mice.

Conclusions/interpretation The findings of this study suggest that the diminution of circulating glycerol reduces endogenous glucose production, contributing to severe fastinginduced hypoglycaemia in leptin-treated rodent models of type 1 diabetes, and support that depletion of glycerol contributes to the glucose-lowering action of leptin.

Keywords Carbohydrate metabolism - Hypoglycaemia . Leptin - Lipid metabolism - Mouse - Type 1 diabetes

Abbreviations
$\begin{array}{ll}\text { HMG } & \text { 3-hydroxy-3-methylglutaryl } \\ \text { RER } & \text { Respiratory exchange ratio } \\ \text { STZ } & \text { Streptozotocin } \\ \text { TAG } & \text { Triacylglycerol }\end{array}$

\section{Introduction}

Administration of the adipose-derived hormone leptin can ameliorate hyperglycaemia and hyperketonaemia in rodent models of insulin-deficient type 1 diabetes, without raising 
circulating insulin levels, and independent of food intake [1-4]. Several mechanisms have been proposed for this glucose-lowering action, including suppressed glucagon and improved insulin sensitivity [1-5], and central leptin therapy was shown to inhibit hepatic glucose production [6] and enhance glucose uptake in brown adipose tissue [7]. With the plethora of metabolic changes that occur with leptin administration in rodent models of type 1 diabetes, the precise mechanism of leptin action in type 1 diabetes remains unclear.

Glycerol is critical in lipid and glucose metabolism; it is the backbone for triacylglycerol (TAG) synthesis, is released along with NEFA during lipolysis and is a substrate for gluconeogenesis. Glycerol infusion at physiological concentrations stimulates glucose output from perfused mouse livers and cultured primary hepatocytes [8], and gluconeogenesis from glycerol accounts for approximately $10 \%$ of hepatic glucose production in type 2 diabetic patients [9]. Elevated circulating glycerol levels are predictive of type 2 diabetes in men [10] and glycerol gluconeogenesis is elevated in rat models of type 1 diabetes [11]. Furthermore, suppressing glycerol gluconeogenesis is a potential therapeutic strategy for treating diabetes, as deletion of glycerol transporters in the liver lowers blood glucose in diabetic $d b / d b$ mice [12], and pharmacological inhibition of lipolysis reduces glycaemia in rats with streptozotocin (STZ)-induced diabetes [13]. Recently, Perry et al showed that the reversal of hyperglycaemia in STZ-diabetic rats by leptin treatment is associated with a reduction in circulating glycerol and glycerol gluconeogenesis [11], suggesting that leptin may reduce glycaemia through glycerol depletion.

To further elucidate the mechanism of leptin-mediated glucose lowering in type 1 diabetes, we analysed the effects of leptin therapy on energy-yielding substrates in mice with STZ-induced diabetes. We found that leptin-treated mice have a severe depletion of hepatic energy-yielding substrates, and that the kinetics of blood glucose normalisation with leptin therapy closely follows the depletion of circulating glycerol, NEFA and TAG. Moreover, the depletion of these substrates is associated with the inability of mice to undergo prolonged fasting, resulting in severe hypoketotic hypoglycaemia, which can be reversed by glycerol administration.

\section{Methods}

Mice C57Bl/6J mice (Jackson Laboratory, Bar Harbor, ME, USA) were housed at room temperature (unless otherwise indicated) with a 12/12 h light-dark cycle with ad libitum access to food (2918, Harlan Laboratories, Madison, WI, USA) and water. All studies used littermate controls. All animal procedures were approved by the UBC Animal Care Committee and performed in accordance with the Canadian Council on Animal Care guidelines.
STZ administration Mice aged 8-10 weeks were injected i.p. with $180 \mathrm{mg} / \mathrm{kg} \mathrm{STZ} \mathrm{(Sigma-Aldrich,} \mathrm{St} \mathrm{Louis,} \mathrm{MO,} \mathrm{USA)}$ dissolved in sodium acetate buffer, $\mathrm{pH} 4.5,5-7$ days before pump implantation.

Leptin therapy Recombinant murine leptin (Peprotech, Rocky Hill, NJ, USA) was dissolved in water and administered by s.c. implantation of osmotic pumps (DURECT Corporation, Cupertino, CA, USA) at a dose of $20 \mu \mathrm{g} / \mathrm{day}$, unless otherwise specified. For leptin injections, leptin was solubilised in water and further diluted to $0.1 \mathrm{mg} / \mathrm{ml}$ in saline $(154 \mathrm{mmol} / \mathrm{l} \mathrm{NaCl})$.

Metabolic assays Measurement of blood glucose and plasma analytes are described in the Electronic Supplementary Material (ESM) Methods. Livers were collected from mice under isoflurane anaesthesia, clamp frozen with liquid $\mathrm{N}_{2}$ and stored at $-80^{\circ} \mathrm{C}$. Hepatic glycogen, glucose, glycerol, TAG and cholesterol measurements are described in the ESM Methods. Hepatic CoA esters were measured by reversed phase HPLC, as described previously [14]. RT-qPCR of hepatic transcripts was performed as previously described [15], with Ppia as a reference gene selected by geNorm analysis, and analysed using the Pfaffl method [16]. Primer sequences are in ESM Table 1.

Body composition and indirect calorimetry Indirect calorimetry, activity and food intake were measured using PhenoMaster metabolic cages (TSE Systems, Chesterfield, $\mathrm{MO}, \mathrm{USA}$ ) at $29^{\circ} \mathrm{C}$. Mice were acclimated to metabolic cages for 5 days before pump implantation and the metabolic run was initiated on day 3 of treatment for $\sim 72 \mathrm{~h}$. Body composition was measured immediately after by dual energy $\mathrm{x}$-ray absorbance (DEXA), using a Lunar PIXImus 2.0 Densitometer (Inside Outside Sales, Madison, WI, USA). Analysis and normalisation procedures are described in the ESM Methods.

Prolonged fasting test Blood glucose/samples were collected immediately before fasting and at indicated times following food removal.

\section{Glycerol and pyruvate tolerance tests}

Mice were fasted for $7 \mathrm{~h}$, and received glycerol $(2.5 \mathrm{~g} / \mathrm{kg}$ dissolved in water), pyruvate ( $2 \mathrm{~g} / \mathrm{kg}$ dissolved in saline) or the corresponding vehicle injection i.p. at time 0 , and blood glucose was measured by glucometer.

Data analysis

Data are expressed as means \pm SEM and were analysed in GraphPad Prism (GraphPad Software, La Jolla, CA, USA). 
Statistical analyses between two groups were performed using a Student's $t$ test, while analyses among three or more groups were performed by one- or two-way ANOVA with a Tukey post hoc test, unless otherwise stated.

\section{Results}

Leptin therapy attenuates metabolic disturbances in STZdiabetic mice STZ-diabetic mice were implanted with s.c. osmotic pumps delivering $20 \mu \mathrm{g}$ /day leptin (STZ-leptin) or vehicle (STZ-vehicle). STZ-vehicle mice were hyperglycaemic, had significantly reduced plasma leptin and insulin levels, and showed a steady decline in body weight relative to non-diabetic controls (Fig. 1a-d). Leptin treatment raised plasma leptin to supraphysiological levels and normalised $4 \mathrm{~h}$ fasting blood glucose over the course of several days, without restoring plasma insulin levels or affecting body weight (Fig. 1a-d). At this dose, leptin fully normalised $4 \mathrm{~h}$ fasted glucose levels, but fed blood glucose levels were only partially reduced (Fig. 1e-f). STZ-vehicle mice displayed polyuria, polydipsia, hyperphagia and decreased locomotor activity, relative to non-diabetic controls, all of which were largely corrected by leptin treatment (Fig. 1g-k). Despite the normalisation of food intake in STZ-leptin mice, pair-feeding studies demonstrate that the metabolic action of leptin therapy is not mimicked by caloric restriction [1-3]. Respiratory exchange ratios (RERs) were downward shifted in STZ-vehicle mice, indicative of enhanced lipid over glucose oxidation (Fig. 11). Interestingly, RERs were partially corrected in STZ-leptin mice. Body composition was altered by STZ administration, and lean mass was modestly reduced by leptin therapy (Fig. 1m), although not when corrected for body weight (data not shown). Collectively, these results show that
Fig. 1 Leptin therapy reverses the disturbances of insulindeficient diabetes. Four hour fasted blood glucose (a), day 8 plasma leptin and insulin $(\mathbf{b}, \mathbf{c})$, and body weight (d) were measured in STZ-leptin (grey circles/bars), STZ-vehicle (white circles/bars) and non-diabetic (black circles/bars) mice, $n=5-8$. Four hour fasted and fed blood glucose levels on days 6 and 8 in a separate cohort, respectively $(\mathbf{e}, \mathbf{f})$. Representative cages showing bedding from singly housed mice (g). Cumulative water intake (h), food intake (i), average energy expenditure (j), average locomotor activity (k), RER (l) and body composition (m). ${ }^{*} p<0.05$, STZ-leptin vs STZ-vehicle; ${ }^{\dagger} p<0.05$, STZvehicle vs non-diabetic; ${ }^{\ddagger} p<0.05$, STZ-leptin vs non-diabetic mice
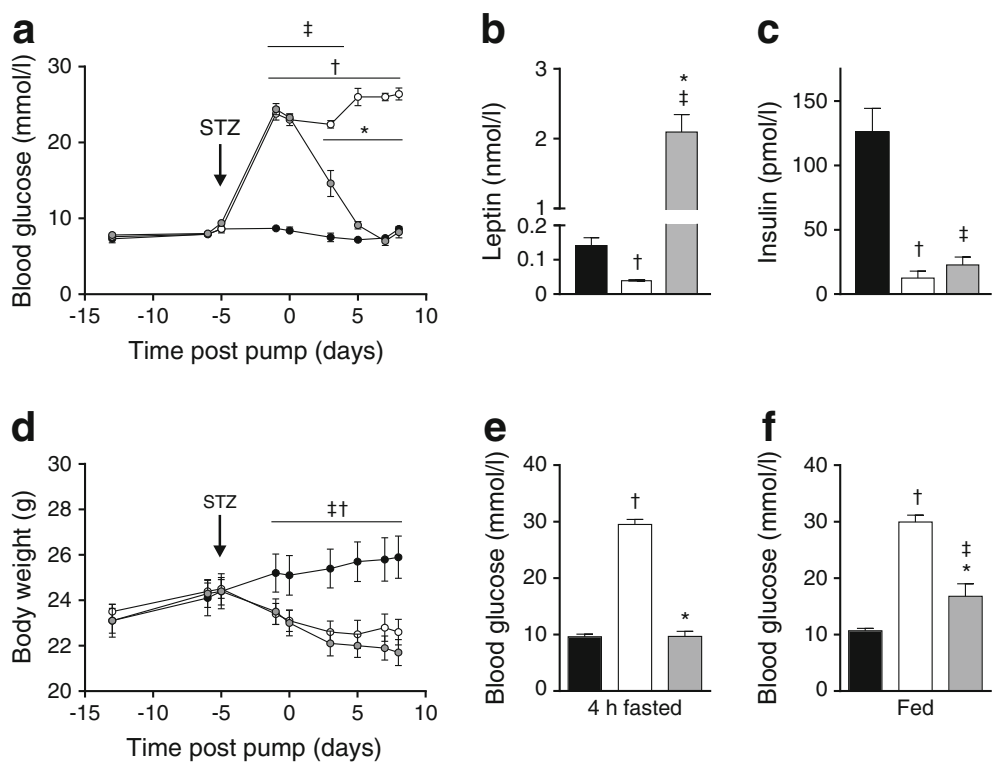

g
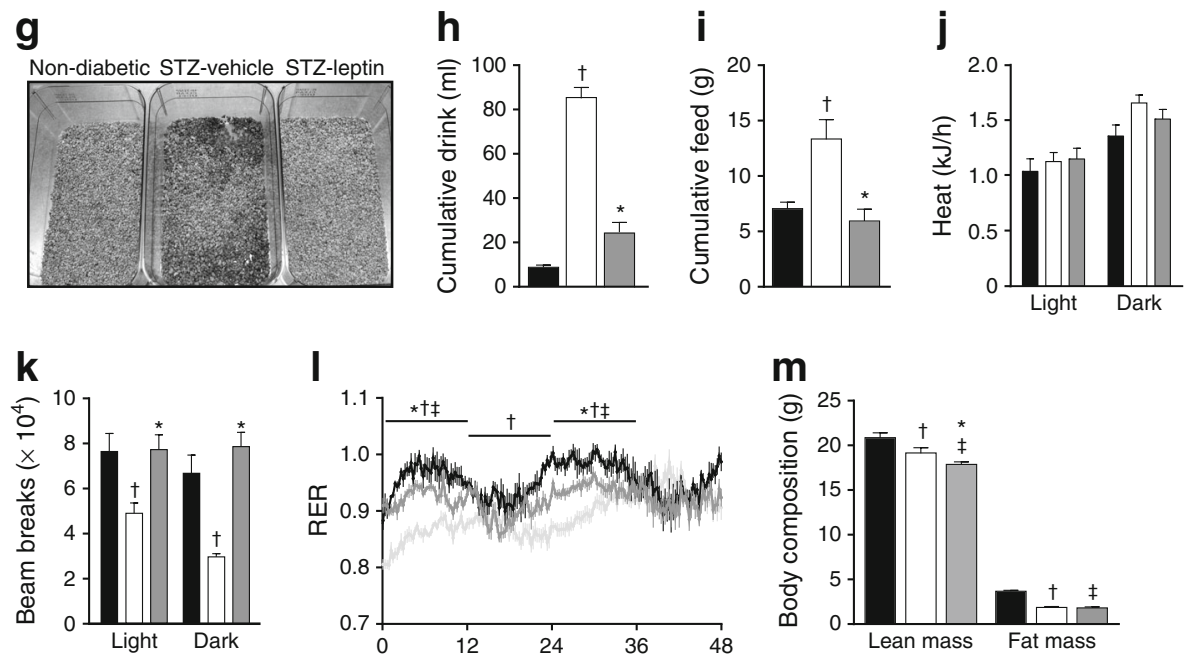

I
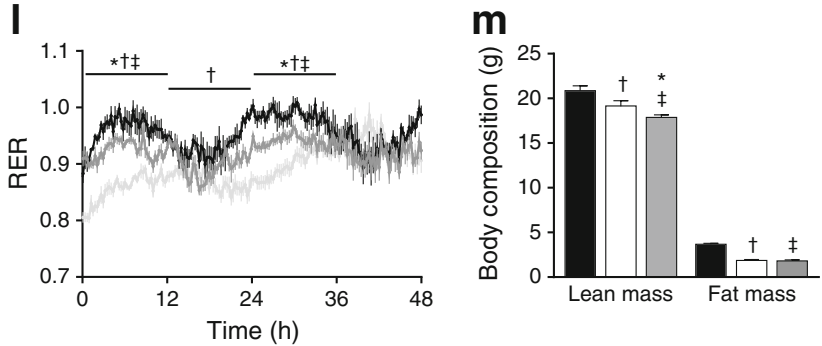
leptin therapy corrects many of the metabolic disturbances of uncontrolled diabetes.

Leptin depletes hepatic energy-yielding stores in STZ-diabetic mice Several studies have shown that leptin reduces endogenous glucose production in rodent models of type 1 diabetes $[1,6,11]$, suggesting that this may drive the glucose-lowering effect of leptin. Therefore, we assessed hepatic energyyielding substrates and gluconeogenic capacity in leptintreated STZ-diabetic mice. Expression of Pck1, the gene encoding phosphoenol pyruvate carboxykinase (Pepck), was elevated in STZ-diabetic mice, but was not reduced by leptin therapy (Fig. 2a). Interestingly, leptin therapy reduced transcript levels of Slc2a2, which encodes the bidirectional glucose transporter Glut2 (Fig. 2a). To elucidate whether gluconeogenesis is inhibited by leptin, we performed pyruvate tolerance tests. Surprisingly, STZ-leptin mice did not have a blunted gluconeogenic response to pyruvate relative to STZvehicle controls (Fig. 2b), suggesting that leptin therapy does not inhibit gluconeogenic capacity. Hepatic glycogen was depleted $\sim 100$ fold in STZ-leptin mice, along with a robust reduction in glucose and TAG content, while hepatic cholesterol was unaltered (Fig. 2c-f). Elevated acetyl-CoA levels in uncontrolled diabetes were reduced by leptin therapy (Fig. $2 \mathrm{~g}$ ) with no changes in free CoA levels (Fig. 2h), indicative of increased hepatic glucose oxidation relative to lipid oxidation, consistent with RER data (Fig. 11) and previous findings [11]. Acetyl-CoA:free CoA ratios (Fig. 2i) were not significantly altered. Hepatic 3-hydroxy-3-methylglutaryl (HMG)-CoA levels were reduced $(p=0.08)$ in STZ-leptin compared with STZ-vehicle mice (Fig. 2j), reflecting a reduction in ketogenesis. Collectively, these data suggest that while inhibition of the gluconeogenic pathway cannot account for the glucoselowering effect of leptin, leptin therapy robustly diminishes hepatic energy-yielding substrates, and normalises ketogenesis and lipid oxidation.

Leptin therapy depletes circulating glycerol and NEFA, concomitant with glucose lowering Observing the depletion of hepatic energy-yielding substrates, we examined whether this corresponded to reduced circulating energy-yielding substrates, and whether this may contribute to the reversal of hyperglycaemia. Leptin therapy gradually normalised blood glucose levels over the course of several days in STZ-diabetic mice treated with leptin; initially, there was a modest decrease in blood glucose levels, and subsequently blood glucose
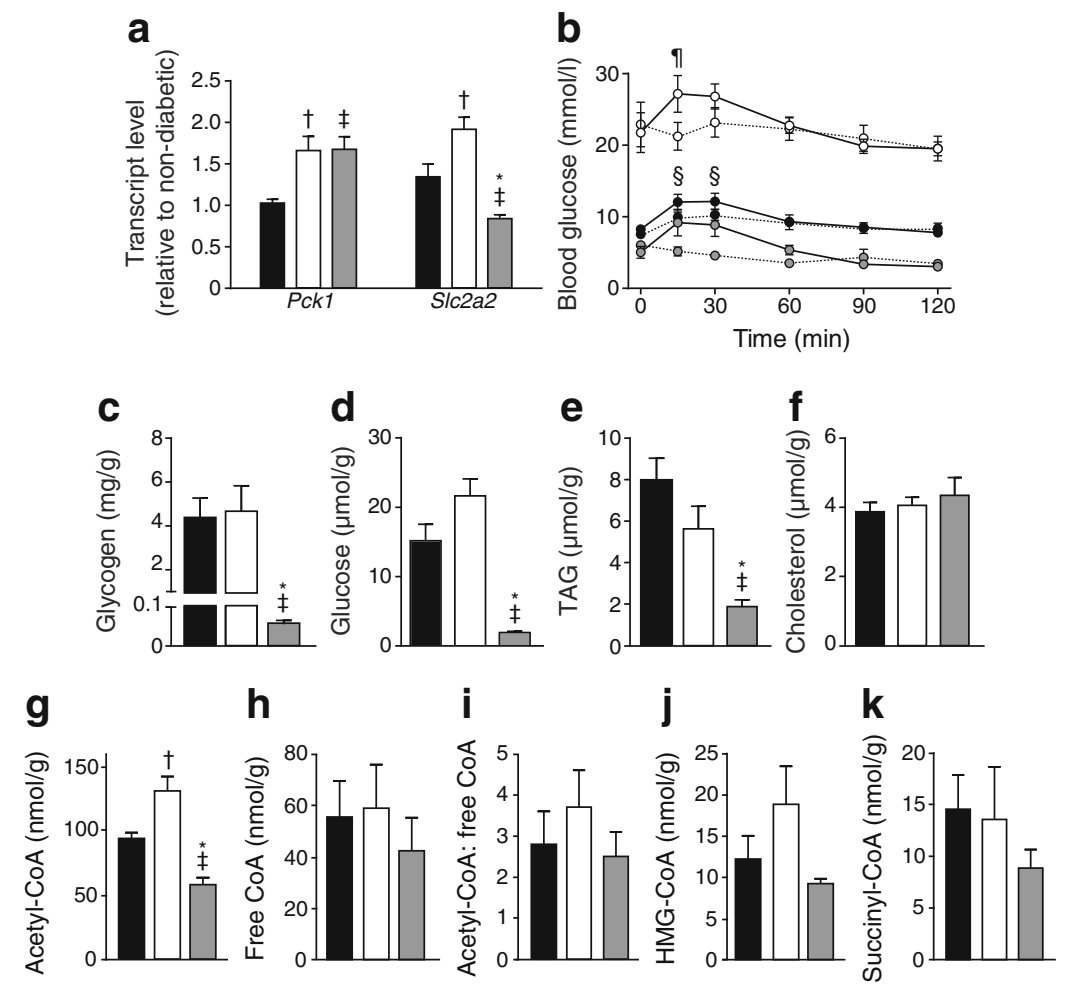

Fig. 2 Leptin therapy depletes hepatic energy-yielding substrates. Livers were harvested following a $4 \mathrm{~h}$ fast on day 8 in STZ-leptin (grey circles/ bars), STZ-vehicle (white circles/bars) and non-diabetic (black circles/ bars) mice (a, c-k). (a) Abundance of hepatic Pck1 and Slc2 2 2 transcripts measured by RT-qPCR, $n=4-6$. (b) Pyruvate tolerance test on day 5 in a separate cohort (pyruvate injection, solid line; vehicle injection, dotted line). Hepatic glycogen (c), glucose (d), TAG (e) and cholesterol (f) content, acetyl-CoA and free CoA levels $(\mathbf{g}, \mathbf{h})$, acetyl-CoA:free CoA ratio (i), HMG-CoA (j) and succinyl-CoA (k), $n=6-8 .{ }^{*} p<0.05$, STZleptin vs STZ-vehicle; ${ }^{\dagger} p<0.05$, STZ-vehicle vs non-diabetic; ${ }^{\star} p<0.05$, STZ-leptin vs non-diabetic; ${ }^{\S} p<0.05$, pyruvate vs vehicle injection in the STZ-leptin group. ${ }^{~} p<0.05$ pyruvate vs vehicle injection in the STZvehicle group 
Fig. 3 The kinetics of hyperglycaemia reversal by leptin correlates with reduced plasma glycerol. Four hour fasted blood glucose (a) and plasma levels of leptin (b), lactate (d), alanine (e), glycerol (f), NEFA (g), $\beta$ hydroxybutyrate $(\beta \mathrm{OH}, \mathbf{h})$, TAG $(\mathbf{i})$ and cholesterol $(\mathbf{j})$, measured daily in STZ-leptin (grey circles), STZ-vehicle (white circles) and nondiabetic (black circles) mice, $n=5-10$. Hepatic glycogen in a matched cohort on day 1 post pump implant, $n=5(\mathbf{c}){ }^{*} p<0.05$, STZ-leptin vs STZ-vehicle; ${ }^{\dagger} p<0.05$, STZ-vehicle vs non-diabetic; ${ }^{\dagger} p<0.05$, STZleptin vs non-diabetic mice

rapidly fell, reaching non-diabetic levels by day 4 (Fig. 3a). This slow normalisation of glucose occurred despite maximal leptin levels being achieved by the first day of leptin therapy (Fig. 3b). Remarkably, $4 \mathrm{~h}$ fasted hepatic glycogen levels were depleted after $24 \mathrm{~h}$ of leptin therapy relative to diabetic and non-diabetic controls (Fig. 3c); however, STZ-leptin mice remained hyperglycaemic until day 4 . We next examined the levels of three circulating gluconeogenic substrates; while there was no lasting effect on plasma lactate and alanine levels (Fig. 3d, e), plasma glycerol levels gradually reduced to $\sim 65 \%$ relative to both controls and initial levels in STZ-leptin mice (Fig. 3f), mirroring the kinetics of glucose lowering. Plasma $\beta$-hydroxybutyrate, NEFA and TAG levels decreased following similar kinetics (Fig. 3g-i). Injection of leptin to induce acute hyperleptinaemia similar to the level achieved by pump implants did not reduce circulating glucose, NEFA, TAG, $\beta$ hydroxybutyrate or glycerol levels compared with vehicleinjected STZ-diabetic controls (ESM Fig. 1). As the regulation of glycerol metabolism by leptin has not been thoroughly described, we also examined glycerol levels in leptin deficient $(o b / o b)$ and leptin receptor deficient $(d b / d b)$ mice (ESM Fig. 2); both models had increased plasma glycerol relative to lean controls, suggesting a role for leptin in glycerol metabolism. Collectively, these findings reveal that the reversal of hyperglycaemia and hyperketonaemia by chronic leptin administration is associated with the gradual reduction of energy-yielding substrates, namely glycerol, NEFA and TAG.

\section{Leptin-treated STZ-diabetic mice are intolerant to fasting We} postulated that the depletion of key energy-yielding substrates by leptin therapy might critically limit endogenous glucose production during fasting. Therefore, we assessed whether STZ-leptin mice could maintain blood glucose levels during a prolonged fast. Following food removal, glycaemia rapidly declined in STZ-leptin mice, reaching those of non-diabetic mice in $2 \mathrm{~h}$, and subsequently progressing to overt hypoglycaemia (Fig. 4a, ESM Table 2). In contrast, nondiabetic and STZ-vehicle controls maintained stable glycaemic levels. In the non-fasted state, plasma glucagon was normalised in STZ-leptin mice (Fig. 4b), consistent with previous reports [2]. In response to fasting, hyperglucagonaemia was diminished in STZ-vehicle mice, potentially indicative of an impaired stimulated-glucagon response as has been shown for STZ-diabetic animals [17, 18],
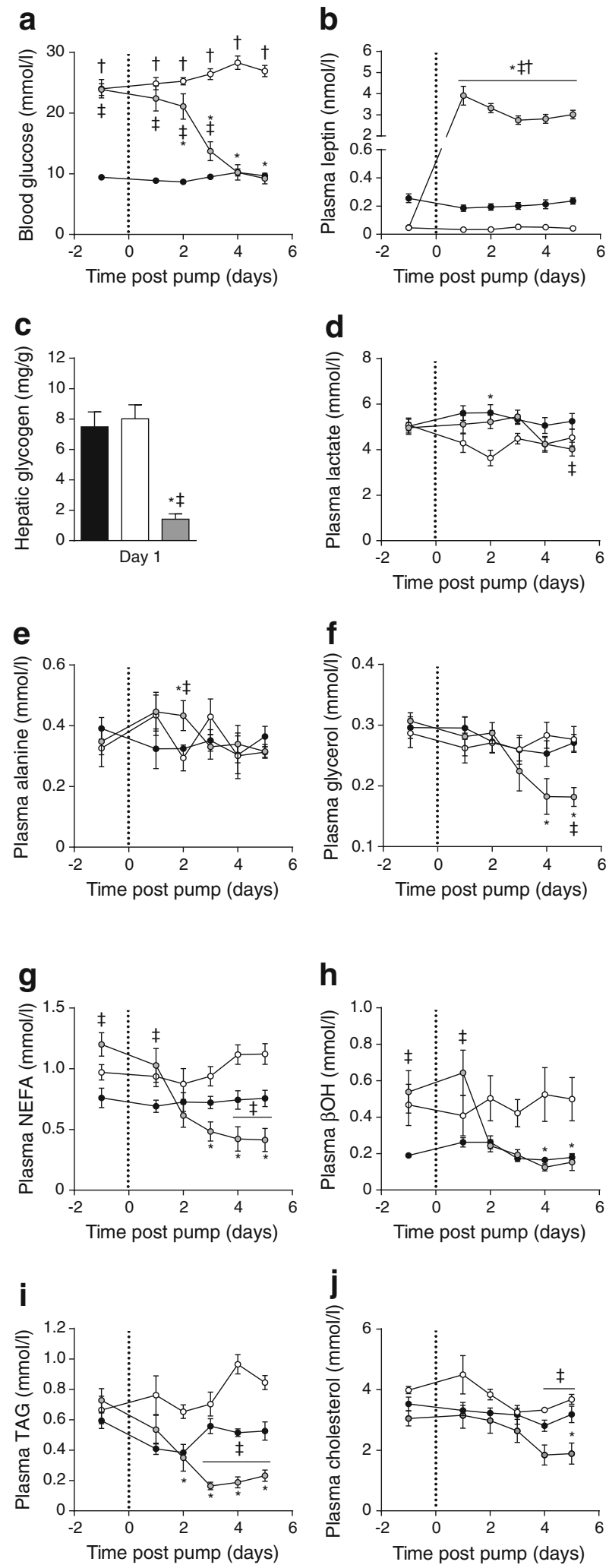

whereas STZ-leptin mice had a non-significant trend towards increased glucagon levels compared with non-fasted levels. As corticosterone is implicated in the glucose-lowering action 
a
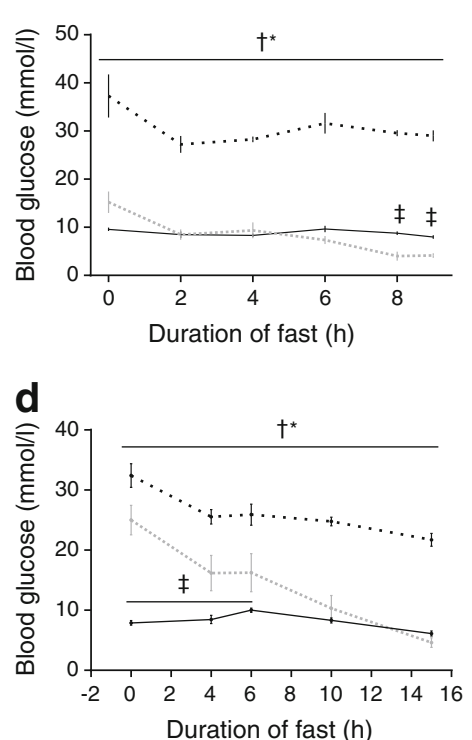

Fig. 4 Leptin-treated STZ-diabetic mice are sensitive to prolonged fasting. STZ-diabetic mice were treated with leptin (grey bars/grey dotted line) at a dose of $20 \mu \mathrm{g} /$ day $(\mathbf{a}-\mathbf{c})$ or $10 \mu \mathrm{g} /$ day $(\mathbf{d}-\mathbf{f})$ and compared with STZ-vehicle (white bars/black dotted line) and non-diabetic controls (black bars/solid black line) on day 7 or 11 of treatment, respectively, $n=4-7$. One STZ-leptin mouse was rescued at $8 \mathrm{~h}$ (a) and $14 \mathrm{~h}$ (d)

of leptin [11], we examined whether suppressed corticosterone levels could account for fasting-induced hypoglycaemia in STZ-leptin mice (Fig. 4c). In the non-fasted state, hypercorticosteronaemia was reversed in STZ-leptin mice, consistent with previously published observations [11]. Interestingly, however, STZ-leptin mice displayed a robust counter-regulatory corticosterone response during prolonged fasting. These data suggest that the hypoglycaemic effect of leptin therapy is independent of plasma corticosterone levels. We repeated the prolonged fast in mice treated with a lower dose of leptin ( $10 \mu \mathrm{g} /$ day $)$, in which hyperglycaemia is more severe in the fed state (Fig. 4d, ESM Table 3). Again, glycaemic levels rapidly declined during fasting in the STZ-leptin group, and three STZ-leptin mice exhibited hypoglycaemia by $15 \mathrm{~h}$. Expectedly, $4 \mathrm{~h}$ fasted $\beta$-hydroxybutyrate levels were normalised in STZ-leptin mice (Fig. 4e), yet, while non-diabetic controls displayed significantly increased $15 \mathrm{~h}$ fasted $\beta$-hydroxybutyrate levels compared with $4 \mathrm{~h}$ fasted levels, this was completely abrogated in STZ-leptin-treated mice. These data are consistent with a robust suppression of endogenous glucose and ketone production. Unlike non-diabetic and STZ-vehicle controls, leptin levels were not reduced after $15 \mathrm{~h}$ of fasting in STZ-leptin mice due to exogenous leptin infusion (Fig. 4f), suggesting that the inability to suppress leptin could be deleterious in prolonged fasting conditions.

Next, we examined the dynamics of energy-yielding substrates between the fed and prolonged fasted $(9 \mathrm{~h})$ states. As
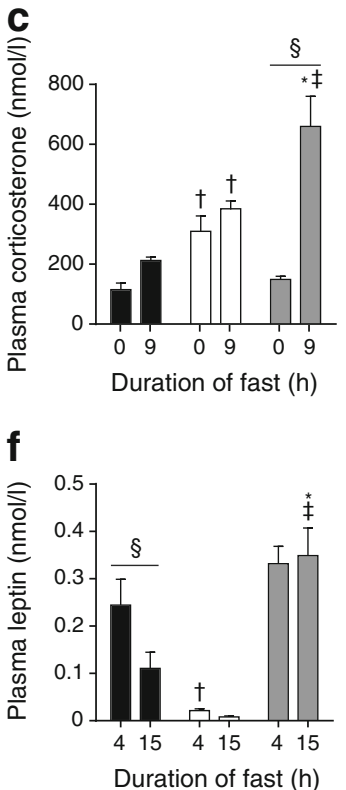

because of hypoglycaemia (blood glucose $<2 \mathrm{mmol} / \mathrm{l}$ ). Plasma glucagon (b), corticosterone $(\mathbf{c}), \beta$-hydroxybutyrate $(\beta \mathrm{OH}, \mathbf{e})$ and leptin $(\mathbf{f})$ were measured at the indicated times. ${ }^{*} p<0.05$, STZ-leptin vs STZ-vehicle; ${ }^{\dagger} p<0.05$, STZ-vehicle vs non-diabetic; ${ }^{\star} p<0.05$, STZ-leptin vs non-diabetic; ${ }^{\S} p<0.05$, between time points

above (Fig. 4e), STZ-leptin mice displayed the expected hypoketonaemia during prolonged fasting (Fig. 5a). Nonfasted glycogen stores were $\sim 60 \%$ lower in STZ-leptin mice compared with STZ-vehicle controls (Fig. 5b), but not depleted to the same extent as seen with $4 \mathrm{~h}$ fasting (Figs. 2c, 3c). In response to prolonged fasting, hepatic glycogen was decreased in all groups including STZ-leptin mice relative to non-fasted levels, revealing that STZ-leptin mice are capable of mobilising hepatic glycogen stores. Plasma lactate (Fig. 5c) and alanine (Fig. 5d) levels were significantly reduced by fasting in all treatment groups, but were not significantly altered by leptin treatment relative to controls. In contrast, fed and prolonged fasted plasma glycerol levels were respectively reduced by $54 \%(p=0.092)$ and $72 \%(p=0.088)$ in STZ-leptin mice, respectively relative to STZ-vehicle mice, although this did not reach statistical significance (Fig. 5e). Correlating with $\beta$-hydroxybutyrate levels, there was a trend towards increased NEFA in non-diabetic mice in response to fasting, whereas STZ-leptin mice had diminished NEFA after extended fasting (Fig. 5f). Fed and fasted plasma TAG levels were also diminished by leptin therapy (Fig. 5g), and plasma cholesterol was modestly decreased (Fig. 5h). These data indicate that fasting-induced hypoglycaemia and hypoketonaemia in leptin-treated mice may be due to insufficient availability of glycerol and NEFA. Interestingly, when leptin was injected to achieve a beneficial effect on glycaemia, and plasma leptin allowed to return to baseline levels following injection, this resulted in rapid repletion of plasma 

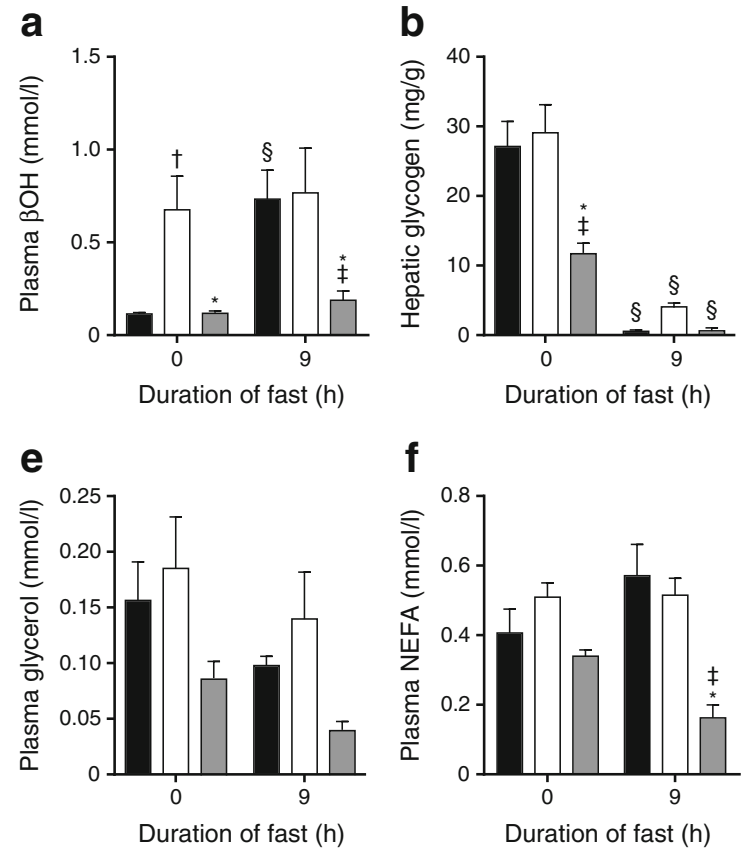

Fig. 5 Gluconeogenic substrate availability is altered during prolonged fasting in leptin-treated mice. On day 7 of leptin therapy, STZ-leptin (grey bars), STZ-vehicle (white bars) and non-diabetic (black bars) mice were killed after 0 or $9 \mathrm{~h}$ of fasting (blood glucose for $9 \mathrm{~h}$ fasted mice in Fig. 4a), and plasma $\beta$-hydroxybutyrate $(\beta \mathrm{OH}, \mathbf{a})$, hepatic glycogen $(\mathbf{b})$,

glycerol; subsequently upon prolonged fasting, STZ-leptin mice were able to maintain steady blood glucose levels (ESM Fig. 3).

Acute glycerol infusion prevents fasting hypoglycaemia in leptin-treated mice To test whether the depletion of glycerol may mediate the hypoglycaemic effect of leptin in the mouse model of type 1 diabetes, we examined whether acute glycerol administration could stimulate gluconeogenesis in STZ-leptin mice and prevent fasting hypoglycaemia. Notably, $4 \mathrm{~h}$ fasted hepatic glycerol content was reduced in STZ-leptin mice relative to controls, consistent with decreased glycerol delivery to the liver (Fig. 6a). Hepatic transcript abundance of Aqp9 (aquaglyceroporin9), which facilitates hepatic glycerol uptake, was also reduced (Fig. 6b). To examine the capacity for glycerol gluconeogenesis in STZ-leptin mice, $7 \mathrm{~h}$ fasted mice were injected with vehicle or glycerol. Expectedly, the STZ-leptin mice injected with vehicle continued progressing towards hypoglycaemia (Fig. 6c). In contrast, glycerol injection acutely raised blood glucose levels, temporarily ameliorating fasting hypoglycaemia in STZ-leptin mice. The glycaemic response to glycerol in STZ-leptin mice is consistent with intact glycerol-fuelled gluconeogenesis. STZ-vehicle and STZ-leptin mice had significantly increased plasma corticosterone and similar plasma glucagon levels following the injection of glycerol (ESM Fig. 4). Taken together with the reduced glycerol gluconeogenic flux reported by Perry et al [11], these data suggest that glycerol depletion contributes to
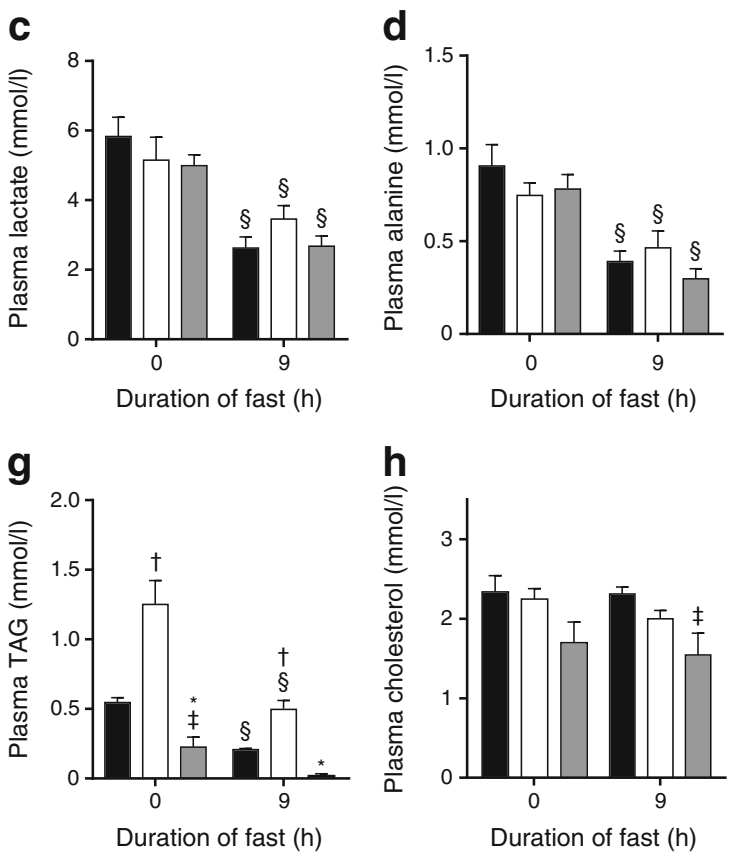

plasma lactate (c), alanine (d), glycerol (e), NEFA (f), TAG (g) and cholesterol (h) were measured, $n=5 .{ }^{*} p<0.05$, STZ-leptin vs STZ-vehicle; ${ }^{\dagger} p<0.05$, STZ-vehicle vs non-diabetic; ${ }^{\star} p<0.05$, STZ-leptin vs non-diabetic; ${ }^{\S} p<0.05$, for same group $0 \mathrm{~h}$ vs $9 \mathrm{~h}$ fasted

leptin-induced hypoglycaemia during fasting in this mouse model of type 1 diabetes.

\section{Discussion}

In the current study, we found that glucose normalisation following leptin therapy in STZ-diabetic mice correlated with the gradual depletion of circulating glycerol, NEFA and TAG. Leptin-treated STZ-diabetic mice rapidly developed hypoketotic hypoglycaemia during prolonged fasting, consistent with reduced gluconeogenesis and ketogenesis. However, STZ-leptin mice were capable of mobilising hepatic glycogen stores (as shown by the reduction of hepatic glycogen before and after fasting), and did not have a diminished gluconeogenic response to pyruvate, relative to controls, indicating that inhibition of glycogenolysis or gluconeogenesis pathways cannot account for the glucose-lowering action of leptin. Leptin-induced hypoketotic hypoglycaemia correlated with depleted plasma glycerol, NEFA and TAG levels, indicative of insufficient substrate availability. Injection of glycerol during prolonged fasting and acute repletion of endogenous glycerol following clearance of injected leptin, ameliorated hypoglycaemia in STZ-leptin mice, suggesting that the gluconeogenic response to glycerol is intact, and that glycerol depletion plays a key role in hypoglycaemia. In parallel, our data are consistent with insufficient NEFA availability for 

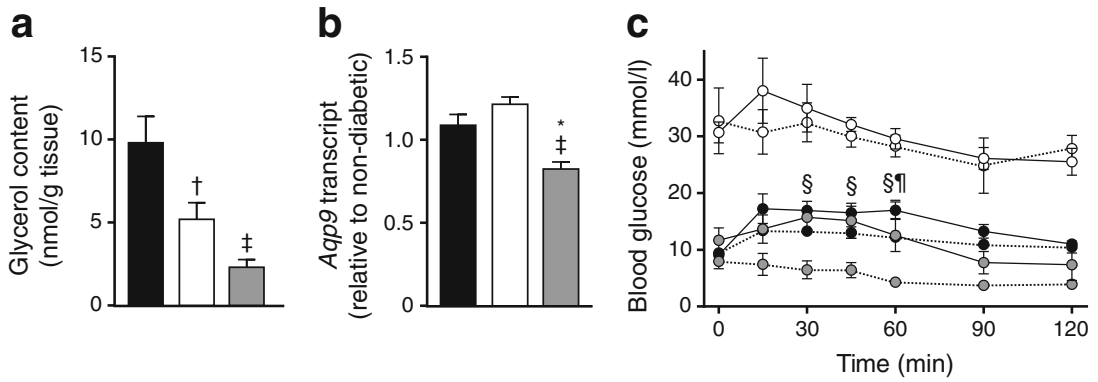

Fig. 6 Glycerol injection prevents hypoglycaemia in STZ-leptin-treated mice. Hepatic glycerol content (a) and transcript abundance of Aqp9 (b) in livers of $4 \mathrm{~h}$ fasted STZ-leptin (grey bars), STZ-vehicle (white bars) and non-diabetic mice (black bars), on day 8 of treatment, $n=6-7$. (c) Mice were injected with either glycerol (solid lines) or vehicle (dotted lines), on day 5 of treatment, $n=4-8$. Statistical analyses in (c) were

oxidation causing fasting hypoketonaemia. Collectively, these data reveal that leptin treatment limits energy substrate availability in STZ-diabetic mice, and that the depletion of glycerol in particular may limit gluconeogenesis resulting in hypoglycaemia during extended fasting.

The inability of leptin-treated STZ-mice to tolerate prolonged fasting is consistent with several other studies showing that leptin therapy decreases hepatic or endogenous glucose production in rodent models of type 1 diabetes $[1,6$, 11]. While leptin therapy suppresses glucagon levels in STZdiabetic mice $[2-5,7,19]$, leptin therapy can reverse hyperglycaemia prior to glucagon suppression [11], and can reverse hyperglucagonaemia without normalising glycaemia in STZ-diabetic rats [5]; this suggests that glucagon suppression alone cannot sufficiently account for the glucoselowering action of leptin. Our finding that, during prolonged fasting, STZ-leptin mice showed a trend towards elevated plasma glucagon, but were hypoglycaemic, supports this notion. Interestingly, although the Perry et al study implicated the suppression of corticosterone in the glucose-lowering effect of leptin [11], we observed a robust elevation of plasma corticosterone during fasting hypoglycaemia in STZ-leptin mice, indicating that the hypoglycaemic effect of leptin during fasting is at least partly independent of corticosterone suppression. Alternatively, leptin can stimulate glucose uptake in the peripheral tissues of rodent models of type 1 diabetes $[6,7]$. However, glucose utilisation in white adipose and some skeletal muscle groups is unaltered in response to leptin [6,7], and other studies report that glucose uptake or disposal is not substantially augmented by leptin therapy $[1,4]$. Considered with the profound fasting intolerance in the current study, these findings suggest that energy-yielding substrate depletion plays an important role in the glucose-lowering action of leptin.

While this study suggests that glycerol depletion contributes to fasting-induced hypoglycaemia in leptin-treated STZdiabetic rodents, its role in the reversal of hyperglycaemia by leptin is less clear. Perry et al showed that leptin infusion in rat models of type 1 diabetes reduced glycerol gluconeogenesis performed by two-way ANOVA with a Sidak post hoc test. ${ }^{*} p<0.05$, STZ-leptin vs STZ-vehicle; ${ }^{\dagger} p<0.05$, STZ-vehicle vs non-diabetic; ${ }^{\ddagger} p<0.05$, STZ-leptin vs non-diabetic. ${ }^{\S} p<0.05$, glycerol vs vehicle injection in the STZ-leptin group. " $p<0.05$ pyruvate vs vehicle injection in the non-diabetic group

by $\sim 90 \%$ [11]. Although this could be achieved by inhibition of the glycerol gluconeogenic pathway by leptin, our data reveal that STZ-leptin mice have reduced hepatic glycerol concentrations (consistent with substrate depletion), and a robust gluconeogenic response to glycerol injection. Moreover, it has been widely reported that leptin therapy takes several days to normalise glycaemia in diabetic rodents $[2-4,6,7$, 19-26], and the current study shows this correlates with the gradual diminution of glycerol levels. Perry et al found that infusion of intralipid with heparin (raising both NEFA and glycerol levels) prevents the glucose-lowering effects of leptin in STZ-diabetic rats [11]. While the Perry study did not test the individual contribution of glycerol and NEFA replacement, our study suggests that glycerol depletion could at least contribute to the hypoglycaemic action of leptin. However, our study also revealed that neither acute glycerol injection nor acute replenishment of endogenous glycerol, fully restored glycaemia to STZ-vehicle levels, indicating that depletion of other energy-yielding substrates (i.e. glycogen, NEFA, TAG), or additional mechanisms, contribute to leptin-induced glucose lowering. Collectively, these observations suggest that glycerol depletion is critical for the hypoglycaemic action of leptin, and that its role in the beneficial glucose-lowering action of leptin in type 1 diabetes warrants further investigation.

Several important concepts emerge in light of the fasting intolerance induced by leptin therapy in STZ-diabetic mice. First, this demonstrates that the level of glycaemic normalisation achieved by leptin is dependent on fasting duration, which should be taken into account when comparing results across studies. Differences in fasting duration could account for discrepancies between studies as to whether physiological leptin doses reverse hyperglycaemia in STZ-diabetic rodents $[5,11,20]$, and why studies examining free-feeding or $2 \mathrm{~h}$ fasted rodents have not reported leptin-induced hypoglycaemia [2, 3, 7, 19]. Second, the hypoketotic hypoglycaemia observed during prolonged fasting suggests that leptin therapy may carry hypoglycaemic risk in type 1 diabetes. We have reported similar profound hypoglycaemic 
effects of leptin in STZ-diabetic mice during insulin tolerance tests [4] and in the recovery phase of glucose tolerance tests [20]. Importantly, when leptin levels were allowed to fall to baseline levels prior to fasting, hypoglycaemia was not observed, corresponding to a lack of glycerol depletion. This suggests that hyperleptinaemia and glycerol depletion are necessary for hypoglycaemia to occur in STZ-diabetes, and that diabetic patients given leptin by injection may suffer hypoglycaemia, depending upon when fasting occurs in relation to leptin injection. Taken together, our study suggests that hyperleptinaemia may cause hypoglycaemia during prolonged fasting due to glycerol depletion, and highlights that additional studies are warranted to fully assess the hypoglycaemic risk of leptin for type 1 diabetes treatment.

Funding This work was supported by the Canadian Institutes of Health Research (CIHR). TJK received a senior scholarship from the Michael Smith Foundation for Health Research. HCD was, and MMK and UHN are, supported by an Alexander Graham Bell Canada Graduate Scholarship from the Natural Sciences and Engineering Research Council of Canada. SMC is a Canada Research Chair and Michael Smith Foundation for Health Research Scholar.

Duality of interest The authors declare that there is no duality of interest associated with this manuscript.

Contribution statement $\mathrm{HCD}, \mathrm{SDC}$ and TJK conceived and designed experiments. HCD acquired and analysed data, and wrote the manuscript. MMK, WLQ and UHN acquired and analysed data. JEK and RWB designed experiments, acquired and analysed data for all HPLC experiments. SK and SMC designed experiments, acquired and analysed data for all metabolic cage experiments. All authors were involved in the discussion and revision of the manuscript, and approved the final version to be published. TJK is the guarantor of this work.

\section{References}

1. Chinookoswong N, Wang JL, Shi ZQ (1999) Leptin restores euglycemia and normalizes glucose turnover in insulin-deficient diabetes in the rat. Diabetes 48:1487-1492

2. Yu X, Park BH, Wang MY, Wang ZV, Unger RH (2008) Making insulin-deficient type 1 diabetic rodents thrive without insulin. Proc Natl Acad Sci U S A 105:14070-14075

3. Wang MY, Chen L, Clark GO et al (2010) Leptin therapy in insulindeficient type I diabetes. Proc Natl Acad Sci U S A 107:4813-4819

4. Denroche HC, Levi J, Wideman RD et al (2011) Leptin therapy reverses hyperglycemia in mice with streptozotocin-induced diabetes, independent of hepatic leptin signaling. Diabetes 60:1414-1423

5. German JP, Wisse BE, Thaler JP et al (2010) Leptin deficiency causes insulin resistance induced by uncontrolled diabetes. Diabetes 59: 1626-1634

6. German JP, Thaler JP, Wisse BE et al (2011) Leptin activates a novel CNS mechanism for insulin-independent normalization of severe diabetic hyperglycemia. Endocrinology 152:394-404

7. Fujikawa T, Berglund ED, Patel VR et al (2013) Leptin engages a hypothalamic neurocircuitry to permit survival in the absence of insulin. Cell Metab 18:431-444
8. Jelen S, Wacker S, Aponte-Santamaria C et al (2011) Aquaporin-9 protein is the primary route of hepatocyte glycerol uptake for glycerol gluconeogenesis in mice. J Biol Chem 286:44319-44325

9. Westergaard N, Madsen P, Lundgren K (1998) Characterization of glycerol uptake and glycerol kinase activity in rat hepatocytes cultured under different hormonal conditions. Biochim Biophys Acta 1402:261-268

10. Mahendran Y, Cederberg H, Vangipurapu J et al (2013) Glycerol and fatty acids in serum predict the development of hyperglycemia and type 2 diabetes in Finnish men. Diabetes Care 36:3732-3738

11. Perry RJ, Zhang XM, Zhang D et al (2014) Leptin reverses diabetes by suppression of the hypothalamic-pituitary-adrenal axis. Nat Med 20:759-763

12. Rojek AM, Skowronski MT, Fuchtbauer EM et al (2007) Defective glycerol metabolism in aquaporin 9 (AQP9) knockout mice. Proc Natl Acad Sci U S A 104:3609-3614

13. Claus TH, Lowe DB, Liang Y et al (2005) Specific inhibition of hormone-sensitive lipase improves lipid profile while reducing plasma glucose. J Pharmacol Exp Ther 315:1396-1402

14. King MT, Reiss PD, Cornell NW (1988) Determination of shortchain coenzyme A compounds by reversed-phase high-performance liquid chromatography. Methods Enzymol 166:70-79

15. Neumann UH, Chen S, Tam YY et al (2014) IGFBP2 is neither sufficient nor necessary for the physiological actions of leptin on glucose homeostasis in male ob/ob mice. Endocrinology 155:716725

16. Pfaffl MW (2001) A new mathematical model for relative quantification in real-time RT-PCR. Nucleic Acids Res 29:e45

17. Weir GC, Knowlton SD, Atkins RF, McKennan KX, Martin DB (1976) Glucagon secretion from the perfused pancreas of streptozotocin-treated rats. Diabetes 25:275-282

18. Kurose T, Tsuda K, Ishida H et al (1992) Glucagon, insulin and somatostatin secretion in response to sympathetic neural activation in streptozotocin-induced diabetic rats. A study with the isolated perfused rat pancreas in vitro. Diabetologia 35:1035-1041

19. Fujikawa T, Chuang JC, Sakata I, Ramadori G, Coppari R (2010) Leptin therapy improves insulin-deficient type 1 diabetes by CNSdependent mechanisms in mice. Proc Natl Acad Sci U S A 107: 17391-17396

20. Denroche HC, Quong WL, Bruin JE et al (2013) Leptin administration enhances islet transplant performance in diabetic mice. Diabetes 62:2738-2746

21. da Silva AA, Tallam LS, Liu J, Hall JE (2006) Chronic antidiabetic and cardiovascular actions of leptin: role of CNS and increased adrenergic activity. Am J Physiol Regul Integr Comp Physiol 291: R1275-R1282

22. da Silva AA, do Carmo JM, Freeman JN, Tallam LS, Hall JE (2009) A functional melanocortin system may be required for chronic CNSmediated antidiabetic and cardiovascular actions of leptin. Diabetes 58:1749-1756

23. do Carmo JM, Hall JE, da Silva AA (2008) Chronic central leptin infusion restores cardiac sympathetic-vagal balance and baroreflex sensitivity in diabetic rats. Am J Physiol Heart Circ Physiol 295: H1974-H1981

24. Lin CY, Higginbotham DA, Judd RL, White BD (2002) Central leptin increases insulin sensitivity in streptozotocininduced diabetic rats. Am J Physiol Endocrinol Metab 282: E1084-E1091

25. Kojima S, Asakawa A, Amitani H et al (2009) Central leptin gene therapy, a substitute for insulin therapy to ameliorate hyperglycemia and hyperphagia, and promote survival in insulin-deficient diabetic mice. Peptides 30:962-966

26. Ueno N, Inui A, Kalra PS, Kalra SP (2006) Leptin transgene expression in the hypothalamus enforces euglycemia in diabetic, insulindeficient nonobese Akita mice and leptin-deficient obese ob/ob mice. Peptides 27:2332-2342 